Progress Report for the period October 15, 1991 to March 14, 1992

\title{
cDNA Expression Map of the Human Genome: Methods Development and Applications Using Brain cDNAs.
}

\author{
Principal Investigator: James M. Sikela, Ph.D. \\ Department of Pharmacology, University of Colorado Health Sciences Center, Denyer, \\ Colorado 80262
}

The following describes progress on human brain cDNA sequencing and mapping that our laboratory has made over the past few months. It should be noted that our first funding installment for the first phase of this grant was obtained approximately two weeks ago. Therefore, the progress that is described represents efforts that were carried out without DOE Genome funds and thus largely are a continuation of pilot studies we began last year. We anticipate, now that DOE funds have arrived, that we will be able to significantly scale up our efforts and productivity.

\section{1) Enrichment for unique cDNAs.}

We have prescreened human hippocampal and fetal brain cDNA libraries with labeled total cDNA and cold human genomic DNA to identify clones that represent abundant mRNAs. By avoiding selection of clones positive hybridizing clones we enrich for those clones that are likely to represent rare mRNAs. The validity of this procedure has been demonstrated by the fact that of the first 137 clones that have been sequenced a duplicate clone was identified only once. No rRNA or mitochondrial clones were found in this collestion. Over 2,000 clones have been selected using this pre-screening protocol.

\section{2) Automated DNA sequencing.}

We have refined methods for rapid single pass sequencing of cDNAs. Currently, single plaques are selected, converted to a Triton-based buffer stock, and CDNA inserts are PCR amplified using vector-specific primers. The PCR products are passed through Centricon 100 membranes to remove primers, dNTPs, etc., and then used directly for automated DNA sequencing on an Applied Biosystems, Inc. (ABI).

We have had excellent results using the dye labeled Universal primer and Cycle sequencing protocol from ABI. Average readable sequence length is approximately $400 \mathrm{bp}$ with an accuracy of $>97 \%$. We have obtained useable sequence information for over $137 \mathrm{cDNAs}$.

\section{3) Database se. Irching.}

Comparison of 137 cDNAs sequences we have obtained has identified only one clone that is a duplicate. Using these cDNA sequences to search Genbank nucleic acid sequences by BLAST has identified several cDNAs that show significant sequence similarity $(>60 \%,>60$ bp) to Genbank entries. Approximately $60 \%$ of the clones appear to represent new genes.

\section{4) Mapping.}

We have utilized sequence information to design PCR primers from the 3' untranslated

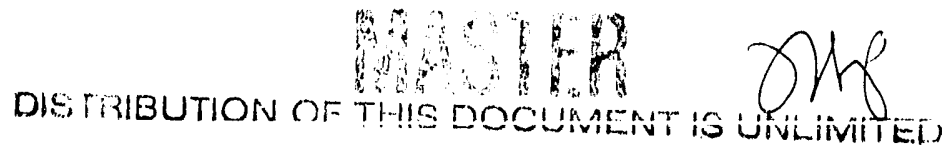


regions of several clones. These have been used for PCR amplification of panels of somatic cell hybrid DNAs to assign these cDNAs to specific human chromosomes.

\section{RESEARCH PLAN FOR 2/92-2/93.}

Specific plans for the coming grant period are as follows:

\section{1) cDNA Libraries and Clone Selection.}

We will continue to use a fetal brain library from Stratagene which we have found to be of sufficient quality to utilize for cDNA collection and sequencing. In addition, we will be receiving human brain cDNA libraries from Dr. Bento Soares. If the libraries are not normalized, we will utilize the same strategy described above for enrichment of unique cDNAs before clones are selected for sequencing.

\section{2) DNA sequencing.}

Because the ABI 373A sequencer we are using is also used for non-genome applications, we will be obtaining another ABI 373 sequencer with partial payment coming from DOE Genome funds. We have also ordered a Perkin Elmer-Cetus 9600 Thermal Cycler that will allow semi-automation of the sequencing reactions. We anticipate that these instruments will allow us to sequence several thousand cDNAs per year.

\section{3) Database Searching.}

We have been utilizing the computer facilities of Dr. Bruce Roe at the University of Oklahoma to perform aspects of the DNA sequence analyses required to do large database searches with cDNA query sequences. So that we do not have to rely on the continued generousity of Dr. Roe, we are requesting supplemental funds (see accompanying pages) to permit us to purchase a SUN SPARC Workstation and necessary accessories to allow our laboratory to independently carry out these analyses. Furthermore, the SPARC format will allow us to utilize a number of software programs specifically designed for cDNA clone analysis (Chris Fields, personal communication). We have talked to Dr. Chris Fields and arranged for one of our laboratory (T.J. Stevens) to spend several days with him at NIH to become familiar with the programs and instrumentation he is utilizing for cDNA analysis.

\section{4) Mapping.}

As a collaboration we will be sending cDNA sequence information to Dr. Mihaelis Polymeropoulos who will be using PCR and somatic cell hybrids to assign CDNAs to specific chromosomes. We are also exploring other mapping strategies including mapping cDNAs by fluorescence in situ hybridization (FISH), and mapping cDNAs to human genomic YACs, which in turn, could be used as FISH probes. Funds for these studies have been requested in other grant applications that are currently under review. Lastly, we have identified a significant number of cDNAs that contain simple sequence repeats. We will be investigating, in collaboration with Dr. Polymeropoulos, how these clones can be used to provide gene-specific polymorphic markers that can be integrated into the human physical and genetic linkage maps. 


\section{Request for Additional Funds for a Sun Sparc IPX Workstation}

As directed by the DOE Human Genome Office (Dr. Marvin Stodolsky) we have included in the budget additional funds to allow us to purchase computer resources that will allow more efficient handling of the cDNA sequence data that we will be generating.

Because we do not have the computing capability to compare sequences locally, Dr. Bruce Roe has been generously allowing us to use the University of Oklahoma's VAX-based GCG programs to reformat and then BLAST cDNA sequences remotely at NCBI. However, we are very limited by both speed of data transmission and by storage allotment on the remote host. When trying to upload sequences for comparison at NCBI we often experience delays of several hours because of modem line problems between our lab and the University of Oklahoma. In addition, during peak demand periods at the University of Oklahoma, response time from the VAX is extremely slow. For these reasons it has become quite clear that the only way we can efficiently analyze and manipulate our cDNA sequences is to carry out these procedures locally.

Over the past few months we have sought advice from Drs. Chris Fields at NIH, Rick Wilson at Washington University, and Bruce Roe about how to best perform these functions within our laboratory. The consensus seems to be that because a workstation would be dedicated to the cDNA sequencing project, we would not need the top of the line instrument, although we would benefit from a UNIX-based system such as Sun Microsystems builds. In the opinion of the above individuals a Sun Sparc IPX (previously sold as the IPC) should meet our needs well. This is the same system that Rick Wilson is presently using to analyze the large amount of genomic and cDNA sequence data he is generating as part of the $C$. elegans genome project.

Therefore, to allow us to purchase this system we are requesting $\$ 11,457$ in additional funds for purchase of a Sun Sparc IPX Workstation with 32Mb RAM, a 424Mb internal hard disk and 16" color monitor. These components were recommended to us by Chris Fields as being quite sufficient to handle the cDNA sequencing data we will be generating. A cost breakdown of the individual components can be found below. With this UNIX-based system, we will be able to analyze our sequences locally using several software packages written for the Sun, including BLAST. In addition, we could more easily trade information with other labs involved in the cDNA sequencing effort, many of which are currently using Suns. The availability of this system will also allow us to take advantage of and potentially contribute to improvements in strategies for automation of sequence analysis. In summary, these capabilities should significantly increase both the speed and effectiveness of our contribution to the cDNA sequencing project.

To facilitate the utilization of the SPARC system for CDNA sequence analysis within our laboratory, I have made arrangements for T.J. Stevens, who presently handles most of our sequence analysis ani has had experience with UNIX systems, to spend several days working with Chris Fields at NIH. During her time there she will be trained by Dr. Fields in handling and analysis of automated DNA sequence information using a Sun Workstation. This experience will greatly facilitate the smooth transition of our laboratory to a UNIX-based system and the rapid integration of the SPARC Workstation into our laboratory's sequence analysis program. 
Sun Sparc IPX:

standard with: $\quad 40 \mathrm{Mhz}$ processor

16MB RAM

207MB SCSI internal drive

1.44MB 3.25" floppy drive

16 " color monitor

Cost (retail) $\$ 13,495$

Add-ons:

16MB additional RAM

$\$ 3,500$

$424 \mathrm{MB}$ additional hard disk memory

$\$ 2,100$

Total:

Less $40 \%$ Univ. of Colorado discount:

$\$ 19,095$

$\$ 11,457$

This amount $(\$ 11,457)$ has been added to the original approved budget of $\$ 151,931$ so that the revised budget is now $\$ 163,388$ for the coming grant period $(3 / 15 / 92-3 / 14 / 93)$.

\section{DISCLAIMER}

This report was prepared as an account of work sponsored by an agency of the United Stat:s Government. Neither the United States Government nor any agency thereof, nor any of their employees, makes any warranty, express or implied, or assumes any legal liability or responsibility for the accuracy, completer iss, or usefulness of any information, apparatus, product, or process disclosed, or represents that its use would not infringe privately owned rights. Reference herein to any specific commercial product, process, or service by trade name, trademark, manufacturer, or otherwise does not necessarily constitute or imply its endorsement, recommendation, or favoring by the United States Government or any agency thereof. The views and opinions of authors expressed herein do not necessarily state or reflect those of the United States Government or any agency thereof. 

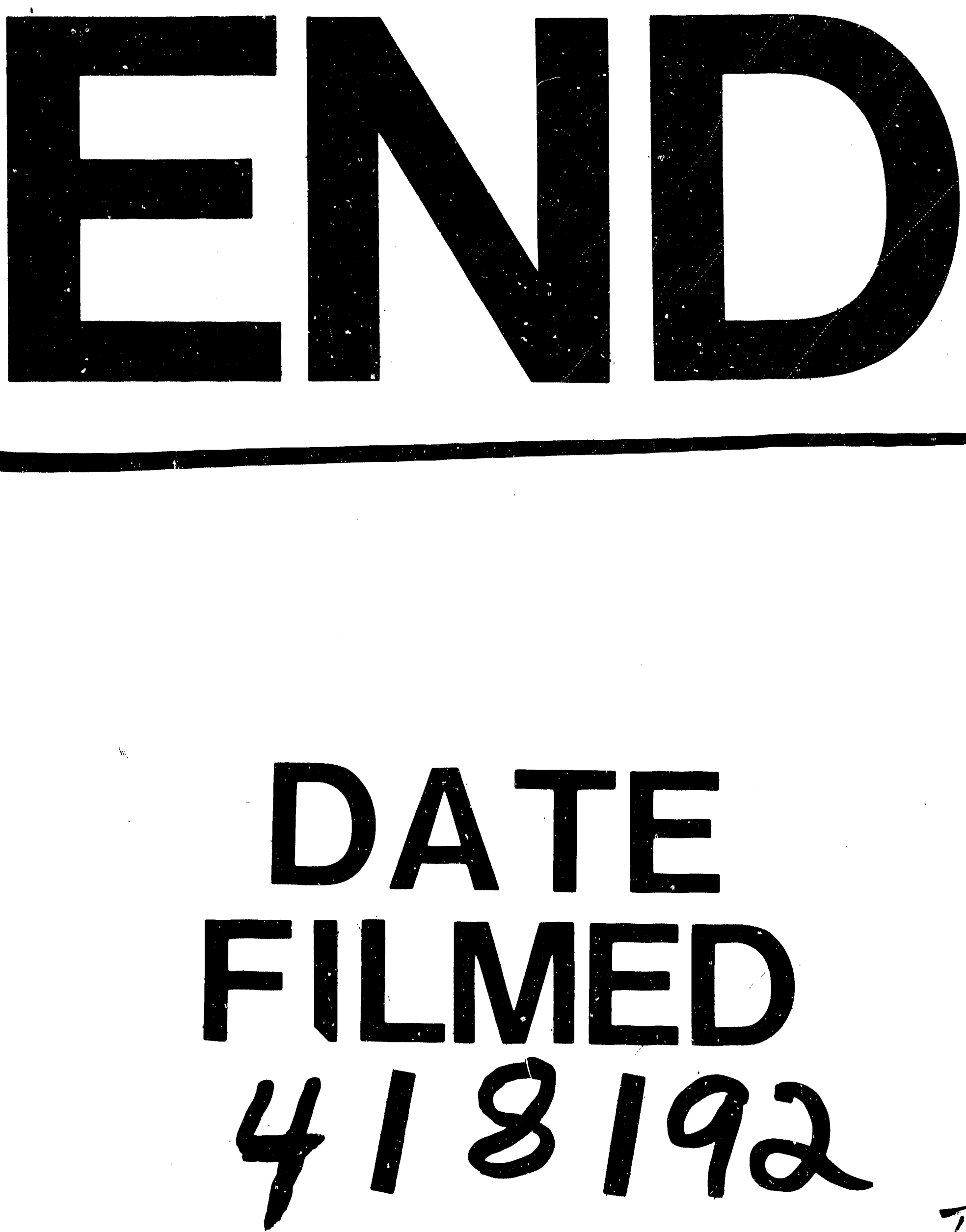
